\title{
MUSIC Algorithm for Respiratory Rate Estimation Using RF Signals
}

\author{
Can Uysal $\mathbb{D}$, Tansu Filik \\ Department of Electrical and Electronics Engineering, Anadolu University School of Engineering, Eskişehir, Turkey
}

Cite this article as: C. Uysal, T. Filik, "MUSIC Algorithm for Respiratory Rate Estimation Using RF Signals", Electrica, vol. 18 , no: 2, pp. 300-309, 2018.

\begin{abstract}
Continuous monitoring of respiratory rate (cycle) during sleep for diseases such as sleep apnea and sudden infant death syndrome (SIDS) can be lifesaving. Wireless radio communications signals are everywhere and can be harnessed for contactless monitoring of the respiratory rates. The amplitude of the received signal strength changes periodically depending on the exhalation and inhalation of the subject. In this paper, subspace-based multiple signal classification (MUSIC) algorithm is applied to estimate the respiratory rate for better results. The proposed method and the other power spectral density (PSD) methods for respiratory estimations are compared with the real laboratory measurements. It is demonstrated that the proposed method estimates the respiratory rate with high accuracy and outperforms the other PSD-based methods which are commonly used in the literature.
\end{abstract}

Keywords: MUSIC, contactless, respiratory rate, wireless signals, software defined radios

\section{Corresponding Author: \\ Can Uysal}

E-mail:

canuysal@anadolu.edu.tr

Received: 08.03 .2018

Accepted: 01.05.2018

(C) Copyright 2018 by Electrica

Available online at

http://electrica.istanbul.edu.tr

DOI: 10.26650/electrica.2018.03405

\section{Introduction}

Continuous monitoring of respiratory rate (cycle) during sleep for diseases such as, sleep apnea, sudden infant death syndrome (SIDS) and chronic obstructive pulmonary disease (COPD) can be life saving [1-3]. When we breathe, air is inhaled into the lungs through the mouth or nose due to muscle contraction and then exhaled by muscle relaxation. The respiratory cycle consists of two parts as inhalation and exhalation. The respiratory rate is defined as the number of respiratory cycles per minute.

There are various methods to continuously monitoring respiratory rate. In literature, these methods can be categorized into two groups: contact-based and contactless. In the contact-based methods, as the name implies, the respiratory rate is estimated via sensors or devices attached to the human body [4]. In clinical environments, for example, capnography that measures the carbon dioxide intensity in the exhaling air via nasal cannula or mask and photoplethysmogram (PPG) which detects the volumetric changes in blood via a sensor which is attached to a finger are commonly used [5,6]. Besides, some methods are developed for the non-clinical environments. For example, respiratory rate estimates are made by analysing the sounds of a person during respiratory via microphones. It is also possible to estimate the respiratory rate by using the strap with accelerometer which is attached to the chest [7-9].

All of these contact-based systems restrict patients' mobility, comfort and sleep patterns. Therefore, contactless respiratory rate monitoring methods have been to developed. Contrary to contact-based methods, there is no need to attach any device or sensor to the human body for the respiratory monitoring in contactless methods. Developed methods can be grouped into two classes: vision based and radio frequency (RF) based. Vision based methods are based on analysis and processing of images [10-12]. These methods require line of sight, depend on daylight, violate the privacy of private life and have high computational complexity. In recent years, electromagnetic RF signals have begun to be used to sense respiratory activity. While RF signals 


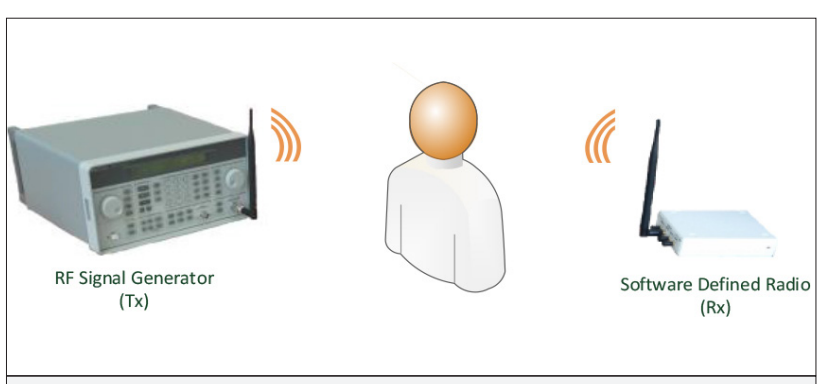

Figure 1. Contactless respiratory monitoring setup

propagate in the air, chest movements of a person whostands on or near the line of sight of the propagation path as shown in Figure 1, affect them. Under this category, Doppler based, ultra-wideband radar-based methods are available [13-17].

In this paper, differently from the above RF based contactless respiratory rate estimation methods, a new method that only uses the received signal strength is proposed. The amplitude of the received signal changes depending on the exhaling and inhaling of the person. In this paper, subspace based Multiple Signal Classification (MUSIC) algorithm is initially applied to estimate the respiratory rate for better results [18]. The proposed MUSIC-based method and the other power spectral density (PSD) methods for respiratory estimations are compared with the real measurement signals collected in laboratory [19-27]. In simulations, it is shown with the various experiments that the proposed subspace-based method outperforms the PSD method with stable and reliable respiratory rate estimation results. This paper is a revised and extended version of a paper that was presented at ELECO-2017 [28]. In this extended paper, in addition to the previous version, some new experimental results including effect of the body orientation, effect of the carrier frequency of the transmitter and the effect of the model order of covariance matrix that is used in MUSIC algorithm are presented in detail. In all these experiments, data lengths are selected same for PSD-based methods and MUSIC algorithm to make a fair comparison. The new experimental results are discussed and concluded. It is shown that subspace based MUSIC algorithm outperforms the all other methods (PSD-based) presented in literature for respiratory rate estimation.

\section{Related Works}

In the existing studies, different types of measurements related to the signal are used. These are Received Signal Strength Indicator (RSSI), Channel State Information (CSI) and raw data from Software Defined Radio (SDR) platform. MAC layer RSSI states the strength of the received signal and has coarsegrained information. As RSSI can take only integer values, high quantization errors can be occur. Compared to RSSI, CSI have fine-grained information and high resolution [29]. CSI consists of the some RF signal propagation effects including the scattering, fading and power decay with the distance [20]. CSI, whose 30 subcarriers are accessed using commodity Wi-Fi cards, in- volves both subcarrier phase and amplitude information. For example, if we deal Wi-Fi signals with subcarriers, RSSI defines a general signal strength value for all subcarriers. However, CSI has individual amplitude and phase information for each subcarrier, examining this quantity leads to results that are more accurate. It is also possible to collect the complex raw signal samples with a SDR platform. In this case, it is possible to get high resolution/rate over the propagation path.

In this study, a SDR platform based system is proposed. The proposed SDR platform based approach can be applied to any RF signals, on the other hand, CSI and RSSI based approaches can only be applied to some specific signals (Wi-Fi signals). Since SDR platform does not have any limitation about frequency selection, bandwidth and transmission mode, the current system can be applicable to any kind of signals.

They utilize RSS measurements taken from many links in a deployed twenty device wireless network. In order to estimate the respiratory rate, power spectral density (PSD) based maximum likelihood estimation is used [19]. They report $0.42 \mathrm{bpm}$ RMS error with 30 seconds of data, which is sufficient for frequency estimates. They also show that using directional antennas improves the system performance. In [20], they propose a new respiratory monitoring system using off-the-shelf Wi-Fi devices which provide CSI parameter. They are able to classify hard conditions like the change of sleeping positions and sleep apnea. They consider two main cases that are respiratory rate estimation under typical sleeping conditions and sleep posture/apnea detection. They compute the Fast Fourier Transform (FFT) of the amplitude of signal segments and the location of the peaks of the FFT in each segment gives the respiratory rate of the breathing person. In [21], they use RSS measurements of a single COTSTX-RX pair but over 16 frequency channels. They report mean absolute error of $0.12 \mathrm{bpm}$ in most realistic scenario. They also estimate the respiratory rate as the peak amplitude of PSD. In this paper, they aim to handle the effect of external motions. Therefore, the Hidden Markov Model (HMM) is used for the motion interference detection. When the posture changes are sensed by the HMM, the system disables the monitoring of the respiratory rate in that time interval. In [22], they utilize a radar technique called as Frequency Modulated Continuous Wave (FMCW) to monitor the respiratory. They propose a method that utilizes the phase information of the complex time-domain signal. They filter the output of the FFT and keep only the peak and its two adjacent samples. Then, they implement the inverse FFT. The phase of the obtained complex time-domain signal will be linear and its slope give the respiratory frequency (rate). In [23], they also track the respiratory rate by using off-the-shelf Wi-Fi devices. The respiratory rate is determined by detecting the location of the peak in PSD of amplitude of CSI measurements. In [24], they extract the hidden breathing signal from noisy Wi-Fi RSS measurements. They achieve the accurate estimates with an Access Point (AP)-device pair. They estimate the instantaneous respiratory rate as the maximum point of the FFT of the RSS measurements. In [25], two USRP 
are used one for the transmitting and one for the receiving the wireless signal at $2.4 \mathrm{GHz}$. They combine four different algorithms, which are zero-crossing, FFT maximum selection, linear predictive coding, least squares harmonic analysis, to estimate the respiratory rate. In [26], they use CSI data provided by commodity Wi-Fi devices. In order to detect the stationary human presence, they take into consideration respiratory of a person as an indicator. The motion interference module first detects whether there is a motion in the environment. If there is no motion, the stationary human detection module performs to find the answer to the question whether there is a human or not. In this section, the respiratory rate that is the location of the peak in the PSD is estimated. If the estimated respiratory frequency (rate) is within the nominal range $(0.1-0.5 \mathrm{~Hz})$, a human presence in the environment is detected. Most of the existing respiratory rate estimation methods in literature use the PSD approach that has many disadvantages although it is a fast method. Since PSD can be easily affected by noise, the probability of error increases. The frequency resolution of PSD is restricted to the number of samples in a measurement window. Therefore, the error value cannot decrease below a certain limit. In addition, it is evaluated that this method is not effective in estimating the respiratory rate of multiple people.

\section{System Overview}

In this section, the basics of respiratory, the effect of inhaling/ exhaling of a person to the received RF signal's strength are discussed. Also the experimental setup used to take real measurements and the measurement model for the respiratory rate estimation are presented.

\section{Basics of Respiration}

Respiration is the process of human's taking oxygen in the air and releasing carbon dioxide to the air to survive. It consists of two phases as inhalation and exhalation as seen in Figure 2. During inhalation, air is inhaled through the mouth or nose and travels towards the lungs. In the meantime, the chest wall expands, the diaphragm contracts and the chest volume increases. During exhalation, this process works in the opposite direction, and it continues cyclically. The processes during inhalation and exhalation are given in Table 1. In a healthy adult the number of breaths per minute is between $12-20,16-22$ in children, and 18-40 in infants.

\section{The Effect of Respiratory to the RF Signal Amplitude}

In this section, the effect of the respiratory to the RF signal amplitude is discussed in detail. The transmitting and receiving antennas are located as shown in Figure 1. The distance traveled by the signals that are reflected (attenuated) from a stationary person on the transmission channel between the transmitter and the receiver changes depending on the breathing of that person. The nominal distance between the transmitter and the receiver is defined as $d_{O}$ and it is expected that the periodic displacement of chest wall changes the traveled distance of the

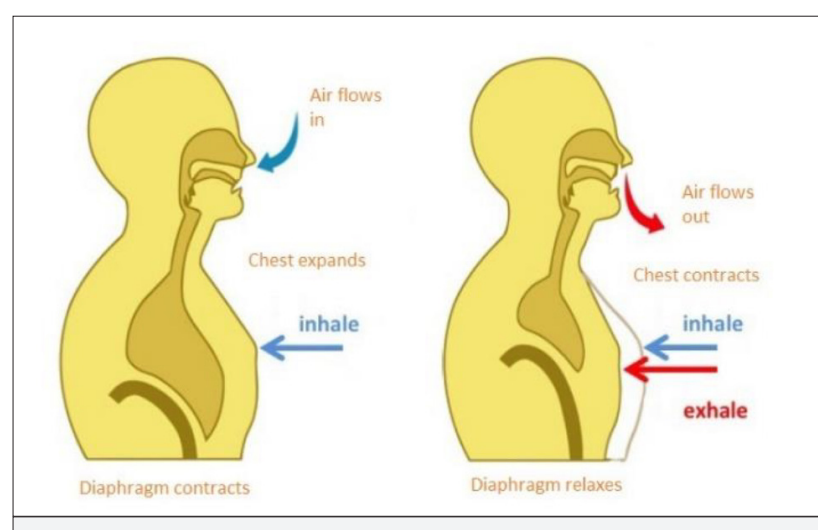

Figure 2. Inhalation and exhalation phases [22]

Table 1. The processes during inhalation and exhalation.

\begin{tabular}{ll}
\hline During Inhalation & During Exhalation \\
\hline -diaphragm contracts & -diaphragm relaxes \\
\hline -chest expands & -chest contracts \\
\hline -air flows in & -air flows out \\
\hline -volume increases & -volume decreases \\
\hline -pressure decreases & -pressure increases \\
\hline
\end{tabular}

reflected signal as periodically [30],

$d(t)=d_{0}+g(t)$

where $d(t)$ is the time-varying distance which is traveled by the signal during the respiratory. Since it is assumed that the displacement of the chest wall is a sinusoidal function of time [31], this distance also varies periodically with the same frequency as the respiratory rate,

$d(t)=d_{0}+g(t)=d_{0}+\Delta A \sin \left(2 \pi f_{R} t\right)$

where $f_{\mathrm{R}}$ represents the respiratory rate, $\Delta A$ is the maximum displacement of the chest wall. The propagation delay according to the traveled distance is defined as follows,

$\tau_{R}(t)=\frac{d(t)}{c}=\frac{d_{0}+\Delta A \sin \left(2 \pi f_{R} t\right)}{c}=\tau_{0}+$ $\tau_{d} \sin \left(2 \pi f_{R} t\right)$

where $\mathrm{c}$ is the speed of light. If the environment is assumed stationary, the time-varying channel impulse response can be modelled as follows,

$h(t, \tau)=\sum_{i} \alpha_{i} \delta\left(\tau-\tau_{i}\right)+\alpha_{R} \delta\left(\tau-\tau_{R}(t)\right)$

where $\alpha_{i}$ is the amplitude value belong to static paths, is the 


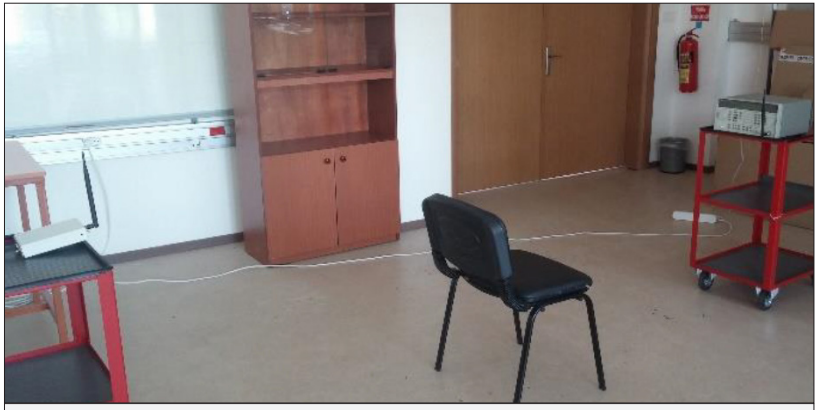

Figure 3. Experimental setup in laboratory

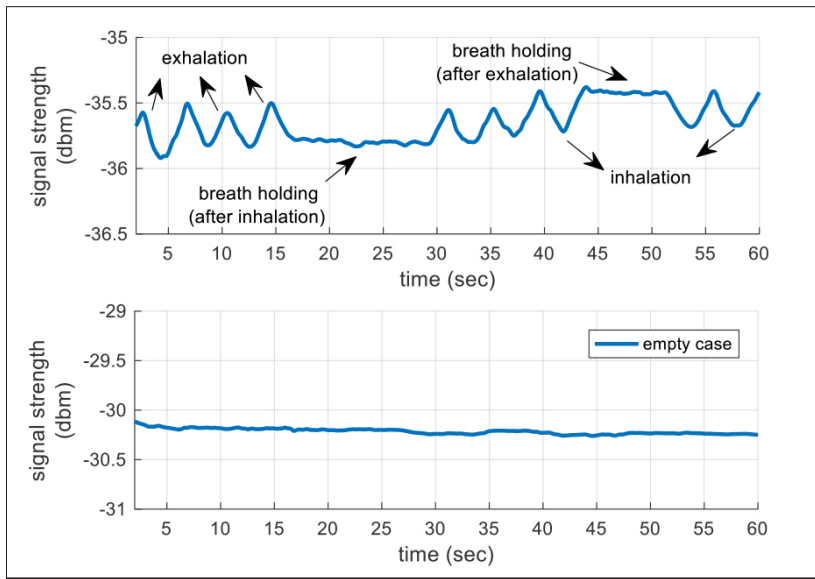

Figure 4. (Top) The effect of the exhalation, inhalation and breath holding on the received signal amplitude. (Bottom) The signal in the absence of the person.

amplitude of the path which is affected by respiratory. $t$ and $\tau$ represent fast and slow varying times, respectively. The received signal is obtained with the convolution of the source signal and the channel impulse response as follows,

$$
\begin{aligned}
& r(t)=s(t) * h(t, \tau) \\
& =\sum_{i} \alpha_{i} s\left(\tau-\tau_{i}\right)+\alpha_{R} s\left(\tau-\tau_{R}(t)\right)
\end{aligned}
$$

As seen from (5), the amplitude of the received signal periodically varies with the same frequency as the respiratory rate.

The displacement of the chest wall along the anteroposterior dimension is approximately $4.2 \mathrm{~mm} \sim 5.4 \mathrm{~mm}$ at the normal breathing and $12.6 \mathrm{~mm}$ at the deep breathing [27], [31]. The chest wall expands outwards when the air is inhaled and contracts inwards when the air is exhaled, as expected. These arguments are also observed and verified with real measurements in our laboratory. The person sitting on the chair in Figure 3 , holds his breath (after exhalation) between the $17^{\text {th }}$ and $30^{\text {th }}$ seconds. Then he continues to breathing until the $43^{\text {rd }}$ second. Afterwards, he holds his breath again (after inhalation) between the $43^{\text {rd }}$ and $52^{\text {nd }}$ seconds. As shown in Figure 4 (Top), these planned movements can be easily monitored with variations on the received signal strength. Figure 4 (Bot- tom) shows the received signal strength in the absence of the person. In this case, the signal level does not show a periodic change. The received RF signal's amplitude level changes with inhaling/exhaling of the person and this causes a periodicity on the received signal due to the breathing movements. Even though the received signal is affected by the ambient noise, it preserves its periodic structure. The respiratory monitoring methods estimate the respiratory rate taking advantage of periodicity of the received signal.

\section{Laboratory Experimental Setup}

In this study, the HP 8647A signal generator is used to generate a continuous wave signal at $900 \mathrm{MHz}$ as the transmitter. Ettus USRP B210 software defined radio is configured as a receiver. USRP (Universal Software Radio Peripheral) is an SDR platform developed by Ettus Research. The experimental setup established for the measurements is shown in Figure 3. A $900 \mathrm{MHz}$ carrier signal with constant amplitude is generated from the transmitter. The power of transmitted signal is adjusted as $0 \mathrm{dbm}$. VERT900 omni-directional vertical antenna with $3 \mathrm{dBi}$ gain is used as an antenna in both transmitter and receiver.

\section{Measurement Model}

Propagation path between the transmitter and receiver is shown in Figure 1. It is assumed that the transmitted signal is a (phase or frequency) modulated signal with a constant peak amplitude which is a reasonable assumption for wireless communication signals. In this case, the averaged received signal strength are assumed constant during communications. On the other hand, the breathing of a person on the propagation path of the signal (as shown in Figure 1,3) will change the amplitude level of the received signal which is already observed in literature $[19,20]$. If there is no movement between transmitter and receiver, the received signal's averaged amplitude should be,

$r(t)=|m(t)|=\mu+\omega(t)$

where $\mu$ is the mean of received signal, $w$ is assumed additive zero-mean noise signal. If a breathing person exists in the environment, the magnitude of the baseband complex signal can be modelled as follows,

$$
\begin{aligned}
& r(t)=\mu+x(t)+\omega(t) \\
& x(t)=A_{C} \cos \left(2 \pi f_{R} t+\phi\right)
\end{aligned}
$$

$A c, f_{\mathrm{R}^{\prime}} \phi$ are the amplitude, respiratory frequency (rate) and phase, respectively. Since breathing requires a periodic action in the form of inhaling and exhaling the cosine model in (8) is suitable to model respiratory. Then the respiratory rate estimation evolves to the frequency estimation $\left(f_{\mathrm{R}}\right)$ of the baseband received signal. 


\section{Respiratory Rate Estimation System}

In this section, the proposed respiratory rate estimation system is discussed. Firstly, the pre-processing steps, which are required to make an accurate estimate are given. Then, the proposed subspace-based MUSIC algorithm is presented in detail. Finally, PSD-based MLE algorithm, which is commonly used in literature is considered.

\section{Pre-processing Steps}

In order to make the respiratory rate estimation algorithm properly with real measurements, some pre-processing steps are required. In this section, the basic pre-processing steps such as Outlier Removal, Downsampling and DC Removal are briefly summarized.

Since the data used is from actual/real measurements, in some cases, due to hardware deterioration several extreme points (spurious peaks) which are not originated from the chest movements can also be observed on the received signal strength. These spurious peaks are refered to as outliers. In Figure 5 (Top), the outliers can be seen that near 8, 10, 38, 46, 55 and 59 seconds. In order to eliminate these spiky peaks (outliers), the well-known Hampel identifier is used [32] in this study. Hampel identifier calculates the median $(\mu)$ and median absolute deviation $(\sigma)$ of the samples in the measurement window. Then, it determines an upper and lower bound using $\mu$ and $\sigma$. Upper and lower bounds are set to $\mu+3 \sigma$ and $\mu-3 \sigma$, respectively. A new sample, which is out of these bounds, is qualified as an outlier. The outliers are detected and removed from the original signal as seen from the Figure 5 (Bottom).

The received signal is sampled by the USRP at $1200 \mathrm{~Hz}$. Since the respiratory rate is below $1 \mathrm{~Hz}$, high sampling rate makes difficult to distinguish the breathing signal in the frequency spectrum. Moreover, high sampling rate increases the com-

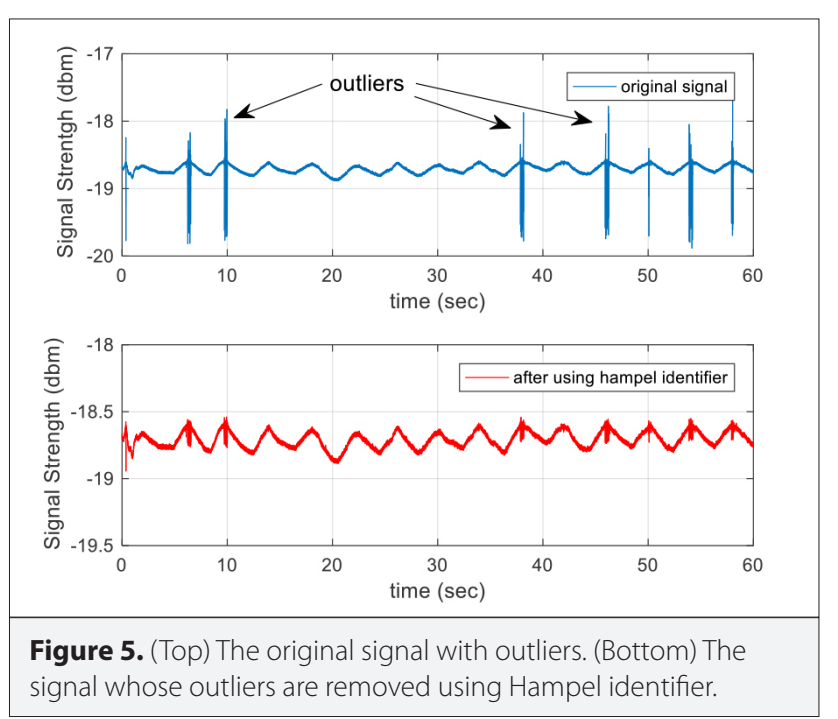

putational cost. Due to these reasons, the received signal is downsampled without distorting its waveform and periodic form. After downsampling, the sampling rate of the received signal is reduced to $1 \mathrm{~Hz}$.

When the spectral analysis is carried out, it is seen that the first component of the signal in frequency domain contains high energy. This DC component that is the average value of the signal in frequency domain suppresses the other frequency components. Besides, DC component does not contain any information about the respiratory. For removing the DC component from signal, the average value is subtracted from entire signal. After DC removal process, the component at $0 \mathrm{~Hz}$ is removed from the frequency spectrum of the signal,

$y(t)=r(t)-\mu$

where is the average amplitude of the received signal and $y(t)$ is the output of the DC removal system.

\section{MUSIC Algorithm}

In this section, we consider the estimation of the respiratory rate using Multiple Signal Classification (MUSIC) algorithm which is a subspace based method [18]. MUSIC algorithm is commonly used to estimate the direction of arrival of signals as well as the frequency of the periodic signals. MUSIC is a super-resolution technique and since it works by separating signal and noise subspaces, it estimates the respiratory frequency more accurate than sample windowed PSD based methods. The $m$ samples collected from the measured and pre-processed signal are modeled as follows,

$$
\begin{gathered}
\mathbf{y}(t)=\left[\begin{array}{c}
y(t) \\
y(t-1) \\
\vdots \\
y(t-m-1)
\end{array}\right]=\mathbf{A x}(t)+\mathbf{w}(t) \\
\mathbf{x}(t)=\left[x_{1}(t) \ldots x_{n}(t)\right]^{T} \\
\mathbf{w}(t)=[\mathbf{w}(t) \ldots w(t-m+1)]^{T}
\end{gathered}
$$

here, $x(t)$ defines the sinusoidal source signals. A matrix contains the sinusoidal source signals and is defined as follows,

$$
\begin{gathered}
\mathbf{a}(f)=\left[\begin{array}{llll}
1 & e^{-j 2 \pi f} & \ldots & e^{-j(m-1) 2 \pi f}
\end{array}\right]^{T} \quad(m \times 1) \\
\mathbf{A}=\left[\begin{array}{llll}
\mathbf{a}\left(f_{1}\right) & \ldots & \mathbf{a}\left(f_{n}\right)
\end{array} \quad(m x n)\right.
\end{gathered}
$$

where $m$ is a positive integer which is the number of samples in the sequence and it also defines the model order of the covariance matrix. $n$ is the number of unknown sinusoidal component. In subspace-based methods, the number of component must be known to decompose the signal and noise subspace. So, it is assumed that $n$ is known. The covariance matrix of $y(t)$ is defined as, 


$$
\mathbf{R}=E\left\{\mathbf{y}(t) \mathbf{y}^{*}(t)\right\}
$$

With the combining of (10) and (13), the covariance matrix becomes,

$$
\begin{gathered}
\mathbf{R}=E\left\{(\mathbf{A} \mathbf{x}(t)+\mathbf{w}(t))(\mathbf{A x}(t)+\mathbf{w}(t))^{*}\right\} \\
=\mathbf{A} E\left\{\mathbf{x}(t) \mathbf{x}^{*}(t)\right\} \mathbf{A}^{*}+E\left\{\mathbf{w}(t) \mathbf{w}^{*}(t)\right\} \\
=\mathbf{A R}_{\mathbf{s}} \mathbf{A}^{*}+\mathbf{R}_{\mathbf{w}}
\end{gathered}
$$

where

$\mathbf{R}_{\mathbf{s}}=E\left\{\mathbf{x}(t) \mathbf{x}^{*}(t)\right\}, \quad \mathbf{R}_{\mathbf{w}}=\sigma^{2} \mathbf{I}$

where $\mathbf{R}_{\mathbf{S}}$ and $\mathbf{R}_{\mathbf{W}}$ are the signal and noise correlation matrices, respectively. $\sigma^{2}$ is the noise power and $\mathbf{I}$ is the identity matrix. The eigendecomposition of $\mathbf{R}$ contains the information on the respiratory frequencies $\left\{f_{k}\right\}_{k=1}^{n}$.

MUSIC is derived from the covariance model in (13) with $m>n$. The eigenvalues of $\mathbf{R}$ matrix are obtained as $\lambda_{1} \geq \lambda_{2} \geq \cdots \geq \lambda_{m}$ and $\left\{s_{1}, \ldots, s_{n}\right\}$ is a set of orthogonal eigenvectors corresponding to $\left\{\lambda_{1}, \ldots, \lambda_{n}\right\}$ and $\left\{g_{1}, \ldots, g_{m-n}\right\}$ are the orthonormal eigenvectors associated with $\left\{\lambda_{n+1}, \ldots, \lambda_{m}\right\}$. The eigenvectors of $\mathbf{R}$ can be divided into two subsets as shown in the following,

$$
\begin{gathered}
\mathbf{S}=\left[\mathbf{s}_{1}, \ldots, \mathbf{s}_{n}\right]_{(m x n)}, \\
\mathbf{G}=\left[\mathbf{g}_{1}, \ldots, \mathbf{g}_{m-n}\right]_{(m x(m-n))}
\end{gathered}
$$

where $\mathbf{S}$ and $\mathbf{G}$ denote signal and noise subspace, respectively. As the noise subspace $\mathbf{G}$ is orthogonal to $\mathbf{A}$ matrix, the following definition can be used,

$$
\mathbf{A}^{*} \mathbf{G}=\mathbf{0}
$$

where $\mathbf{A}$ is a function of the frequencies $\left\{f_{k}\right\}_{k=1}^{n}$. The columns of $\mathbf{G}$ belong to the null space of $\mathbf{A}$ as shown in (17). The true respiratory frequencies $\left\{f_{k}\right\}_{k=1}^{n}$ are the only solutions of the equation $\mathbf{a}^{*}(f) \mathbf{G G}^{*} \mathbf{a}(f)=0$ for any $m>n$. The MUSIC algorithm is defined in two steps as follows,

- Step-1: Compute the sample covariance matrix

$$
\widehat{\mathbf{R}}=\frac{1}{N} \sum_{t=m}^{N} \mathbf{y}(t) \mathbf{y}^{*}(t)
$$

and its eigendecomposition. $\hat{\mathbf{S}}$ and $\hat{\mathbf{G}}$ are the signal and noise eigenvectors obtained from matrix.

- Step-2: Using $\hat{\mathbf{G}}$ noise subspace which is obtained in Step1 determine the respiratory frequency estimates as the locations of the $n$ highest peaks of the estimation function
$\widehat{P}_{\text {music }}(f)=\frac{1}{\mathbf{a}^{*}(f) \widehat{\mathbf{G}} \widehat{\mathbf{G}}^{*} \mathbf{a}(f)}$

In this study, since there is a single patient, the number of sinusoidal signals is assumed one $(n=1)$. It is also possible to monitor multiple patients $(n>1)$ with the MUSIC algorithm.

\section{PSD-Based MLE}

In this section, the power spectral density (PSD)-based maximum likelihood estimation (MLE) is given as an extension of the classical sinusoidal parameter estimation problem [19]. In [19], a respiratory rate estimator which estimates the respiratory rate as the frequency at the maximum of the PSD is proposed. The MLE of frequency $\hat{f}_{R}$ is defined as,

$\hat{f}_{R}=\underset{f_{\min } \leq f \leq f_{\max }}{\operatorname{argmax}}\left|\sum_{t=1}^{N} y(t) e^{-j 2 \pi f t}\right|^{2}$

In the power spectrum, the frequencies close to zero are excluded specifically. They determine the sampling rate to satisfy the Nyquist criterion.

\section{Experimental Results}

In this part, we design some experiments in laboratory in order to observe the performance of the respiratory monitoring system. In order to get statistically significant performance results, a total of 270 measurements (trials) are collected in laboratory. In order to analyse the effect of carrier frequencies and body orientations of a participant to respiratory rate estimation, the distances between the transmitter and the receiver, the transmit powers and carrier frequencies are changed in a controlled manner while collecting data. Each measurement is one and half and three minutes long. In all these measurements, the distances between the transmitter $\&$ subject and subject \& receiver are taken equal, as the participants are sitting the midpoint of the distance as shown in Figure 3. The different length sliding window is shifted along the signal and the respiratory rate is estimated from each measurement window. The proposed subspace-based MUSIC method is first time applied to estimate respiratory rates using received signal strength. The mean absolute error (MAE) is used as a performance metric, which measures the accuracy of the system. The participants try to keep their respiratory rates fixed using a chronometer.

In order to analyse the effect of selected window length, transmitter frequency and body orientations of a participant to respiratory rate estimation, different experiments are carried out. For all these different experiments, the proposed subspace based MUSIC method are compared with the commonly used PSD-based MLE method in [19]. 

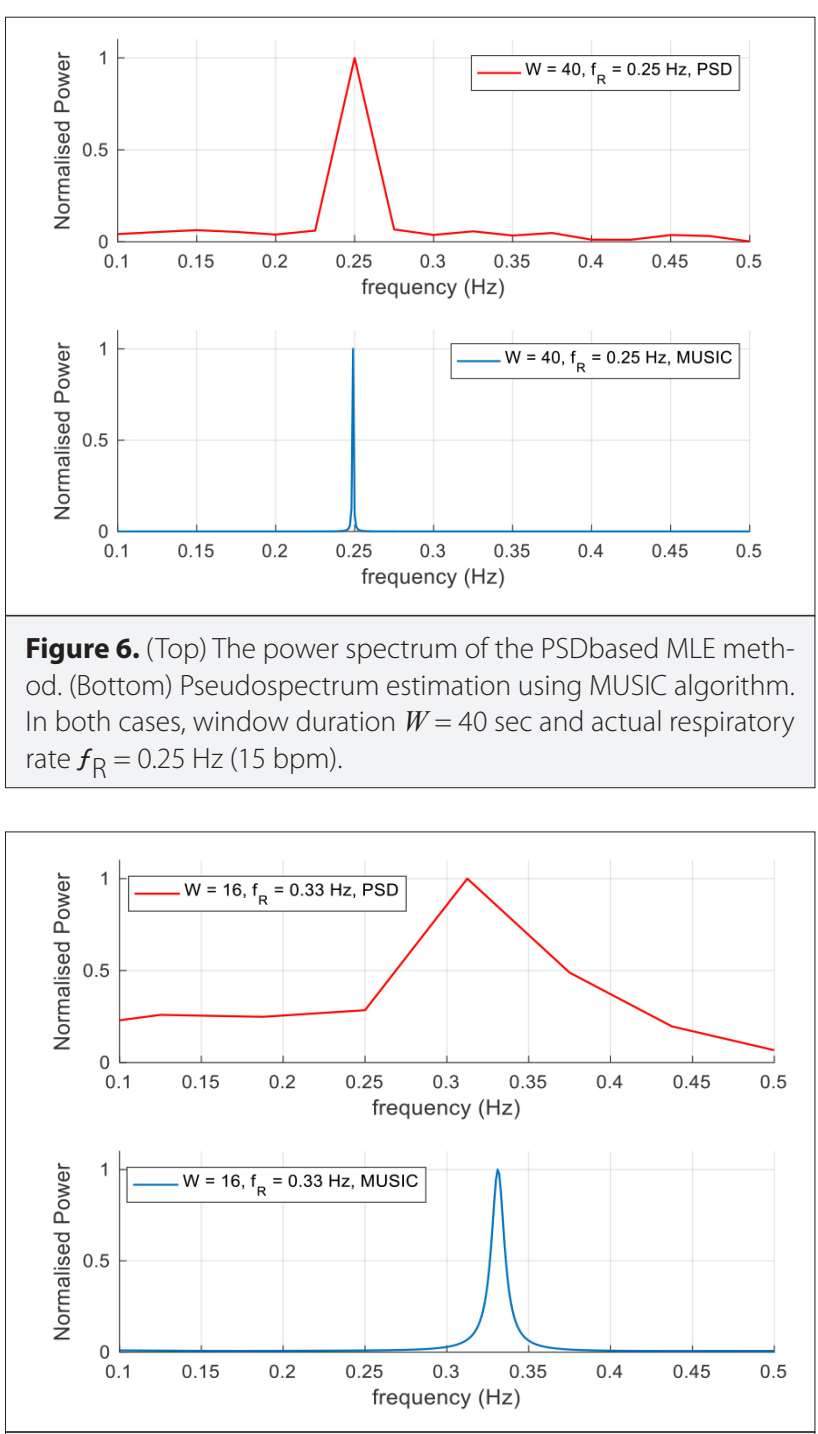

Figure 7. (Top) The power spectrum of the PSD-based MLE method. (Bottom) Pseudospectrum estimation using MUSIC algorithm. In both cases, window duration $W=16 \mathrm{sec}$ and actual respiratory rate $f_{R}=0.33 \mathrm{~Hz}(20 \mathrm{bpm})$.

In Figure 6, normalised spectrum estimations, which are obtained using MUSIC and the PSD algorithms, are compared. The number of samples (window durations) are selected as $W=40$ seconds for these two algorithms. In this experiment, actual respiratory rate is $15 \mathrm{bpm}(0.25 \mathrm{~Hz})$. As seen from the figure, the peaks in both spectrums occur at the frequencies corresponding to the respiratory rate of the person. It can be seen that the peak in the MUSIC spectrum has narrower (sharp) main lobe width according to PSD spectrum. As expected, it is observed that the MUSIC spectrum has higher frequency resolution than the PSD spectrum that is commonly used in respiratory rate estimation literature.

In Figure 7, the window duration is decreased to $W=16 \mathrm{sec}-$ onds and the actual respiratory rate is $20 \mathrm{bpm}(0.33 \mathrm{~Hz})$. In this

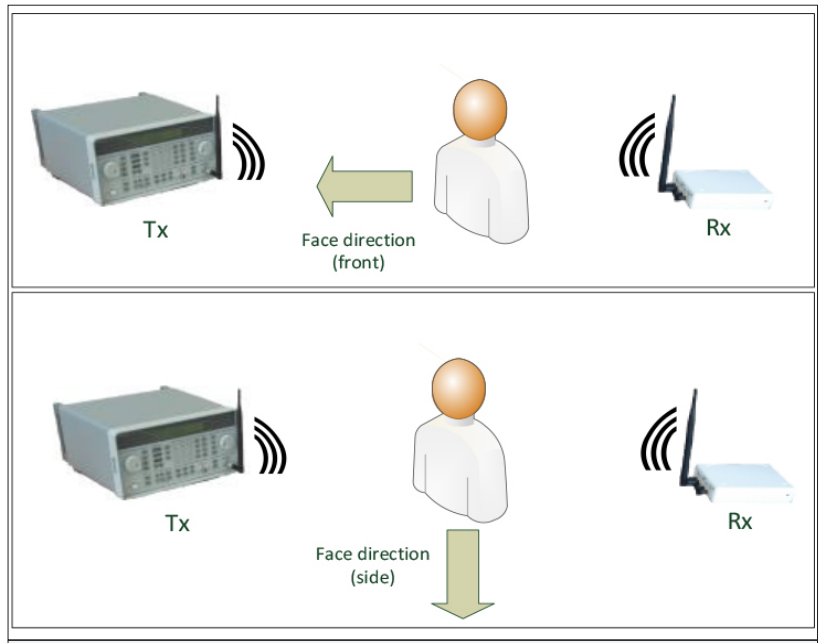

Figure 8. The positions of the user during body orientation experiments. Top and bottom figures show position-1 and position-2, respectively.

case, although the main lobe width of the peak at the MUSIC spectrum expands, it is not significantly effected by the decrease in window duration. However, it can be seen that the main lobe width increases excessively in the PSD spectrum. It can be seen that the frequency resolution of the PSD decreases with the shortening of window duration. In the case of low signal to noise ratio (SNR), it is evaluated that the MUSIC algorithm will be more accurate and robust. Besides, in multiple person case, PSD-based method cannot distinguish different frequencies due to its low resolution.

\section{Effect of the Body Orientation}

In the first experiment, the participant is sitting on the LOS between the transmitter and the receiver as in position-1 (front). In second experiment, the participant swaps his position to the position-2 (side). The positions are shown in Figure 8. In Figure 9, the estimation accuracies of the proposed MUSIC and PSD based MLE methods for $W=20$ are shown. It is shown in the figure that better performances are obtained in case of position-1. Fig. 10 shows the effect of the body orientations relative to the transmitter (front and side) on the estimation performances of the subspace-based MUSIC method and PSD-based MLE method. In both positions of the participant, it can be seen that all methods can estimate the respiratory frequency. During respiration, the displacement of the chest wall is $4.2 \sim 5.4 \mathrm{~mm}$ in the anteroposterior dimension and $0.6 \sim 1 \mathrm{~mm}$ in the mediolateral dimension [27], [31]. Therefore, as the change in the anteroposterior dimension is greater than the change in the mediolateral dimension, with more variation in the amplitude level of the received signal is observed in this case. The obtained greater variation reveals that more distinct sine wave and better performances are obtained in case of position-1.

In addition, it can be seen in the figure, error rates decrease with the increasing of window duration. However, selection of the 


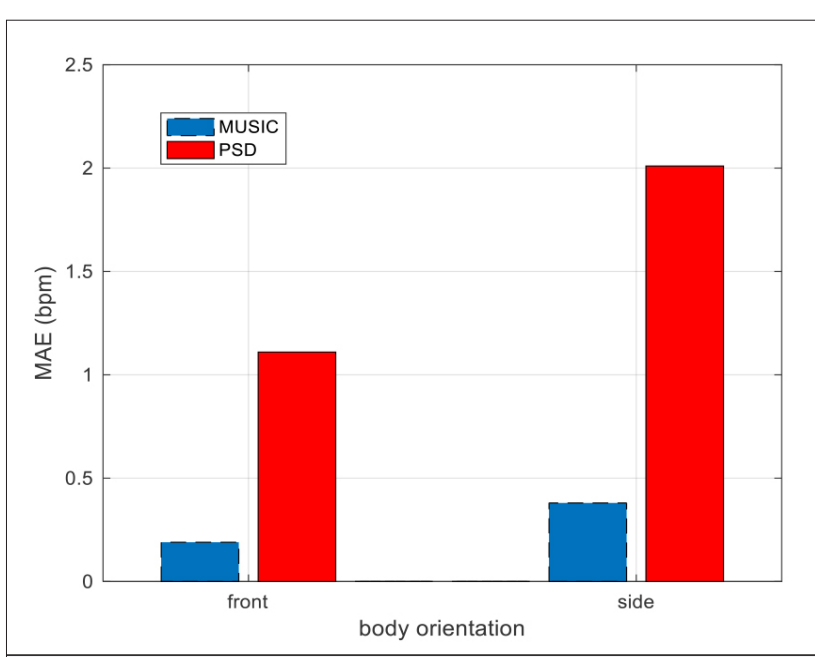

Figure 9. The effect of body orientation for $W=20$

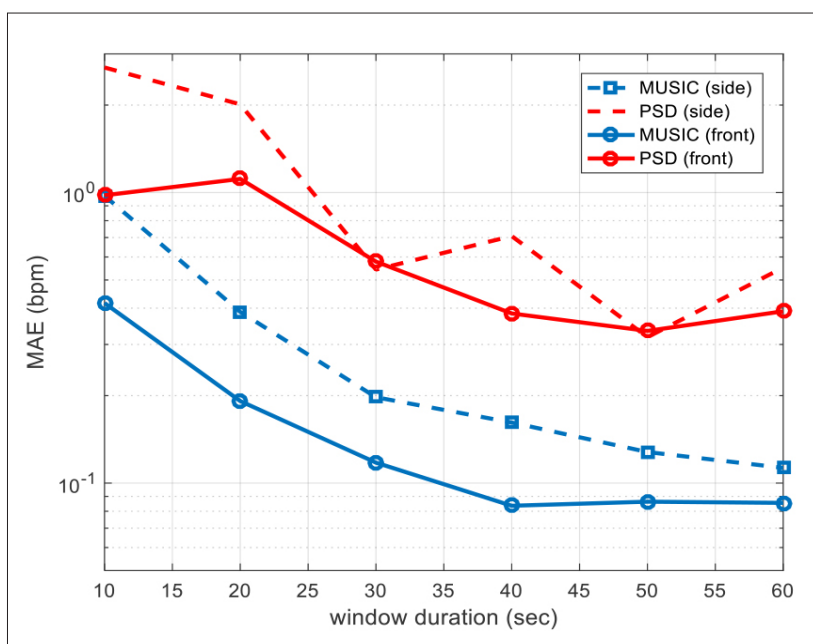

Figure 10. Performance comparison between PSD and MUSIC methods according to the $W$ in the body orientation experiment.

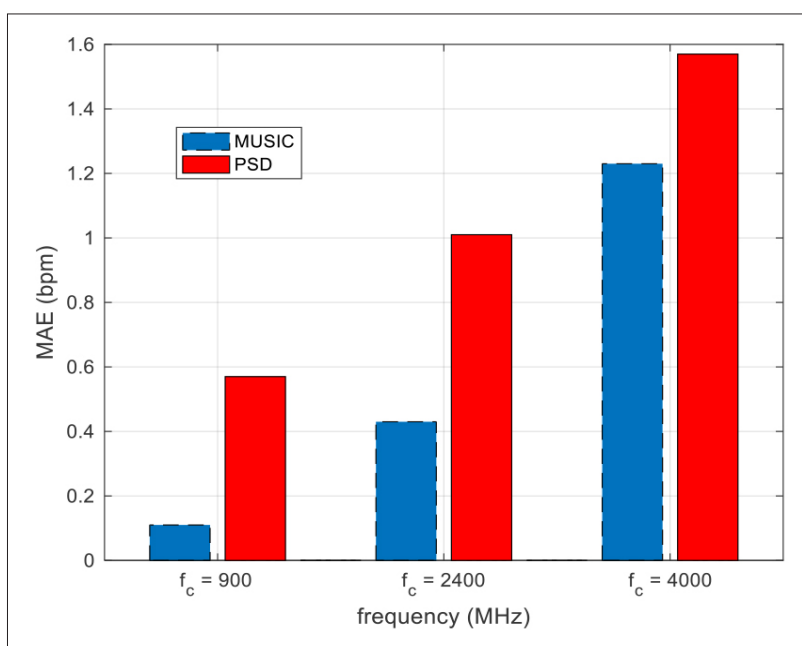

Figure 11. MAE (bpm) values according to the carrier frequencies.

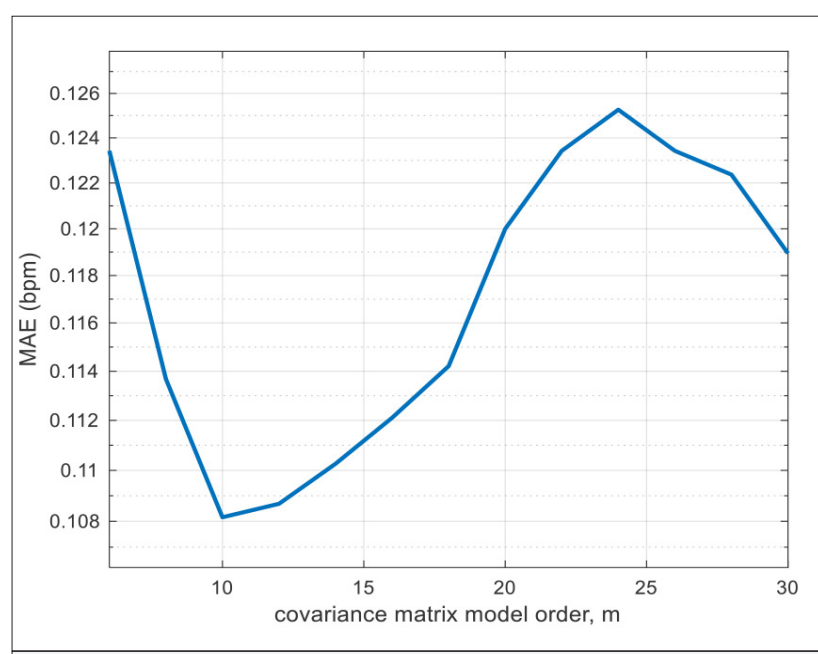

Figure 12. Respiratory rate estimation performance of MUSIC method according to the covariance matrix model order, $W$ is selected $40 \mathrm{sec}$

long window duration reveals some disadvantages. We take the samples from the received signal for a duration $W$ before estimating the respiratory rate. Therefore, window duration $W$ is important because it determines the waiting time. Moreover, the long window duration causes to miss sudden changes in respiratory rate. The proposed MUSIC based method outperforms the PSD-based MLE method for all window durations. As seen from the Figure 10, while the MAE of the proposed method is lower than 0.1 breaths per minute (bpm) for all window durations $W$ $>30$ seconds, the PSD-based method cannot reach this rate for all window durations. Especially for short window duration ( $W$ $<30$ ), the performance of our proposed method is quite better than the PSD-based MLE method. This is important for the respiratory monitoring system requiring low latency.

\section{Effect of the Carrier Frequency}

This experiment is designed to show the effect of the carrier frequency of the transmitted signal to the estimation performances. Figure 11 shows the MAE values in terms of bpm of different carrier frequencies for $W=30$. Carrier frequencies of the transmitter are set to $900 \mathrm{MHz}, 2.4 \mathrm{GHz}$ and $4 \mathrm{GHz}$, respectively. Distance between the transmitter and the receiver is specified as 2 meters and the transmitted power is adjusted as $0 \mathrm{dBm}$. We observe that respiratory rate estimation accuracy decreases with the increasing of the frequency of the radio signal. This is because the effect of human respiratory on radio signal propagation decreases as the frequency of the radio signal increases. As seen from the Figure 11, the best performance is obtained when the carrier frequency is selected as $900 \mathrm{MHz}$. In this case, the proposed MUSIC method can estimate the respiratory rate with $0.1 \mathrm{bpm}$ accuracy. The lowest accuracy is obtained with PSD-based method for all carrier frequencies. 


\section{Effect of the Covariance Matrix Model Order}

Figure 12 shows the effect of the covariance matrix model order $(m)$ to the respiratory rate estimation accuracy $m$. corresponds the number of samples in the sequence which are used to construct the covariance matrix. As the subspace-based methods use the eigendecomposition of the covariance matrix, the model order is a critical parameter. The sliding window duration is set to 40 seconds. It can be seen from the figure, the highest accuracy is obtained when $m$ is selected as 10 . Selecting a large value for $m$ not only reduces the system accuracy but also increases the computational complexity because the size of the matrix increases.

\section{Conclusion}

In this study, we present a contactless respiratory monitoring system that requires no devices or sensing module on the human body. The proposed system uses subspace based MUSIC algorithm to estimate the respiratory rate using only a single TX/RX pair. In the laboratory, we designed some experiments using real measurements to show the performance of the proposed respiratory rate estimation system. The proposed system uses complex raw data collected with SDR platform that does not any limitation about frequency selection, bandwidth, etc. In the experiments, many cases including the effect of carrier frequency, the effect of the covariance matrix model order, the effect of the body orientation are investigated. Our proposed MUSIC-based respiratory rate estimation method is compared with the PSD-based MLE method for the different measurement window durations and scenarios. It is shown with several experiments that the proposed MUSIC-based method can estimate the respiratory rate of a person with $0.08 \mathrm{bpm}$ mean absolute error in ideal case. It is also shown that the MUSIC method outperforms the commonly used PSD-based MLE method. The performances of the MUSIC algorithm, which provide more accurate estimates with low signal strength and limited number of samples, are shown through real measurements.

Peer-review: Externally peer-reviewed.

Conflict of Interest: The authors have no conflicts of interest to declare.

Financial Disclosure: This work was supported by Anadolu University Scientific Research Projects Fund with projects 1705F416.

\section{References}

1. M. Younes, "Role of respiratory control mechanisms in the pathogenesis of obstructive sleep disorders", J Appl Physiol (1985), vol. 105, no. 5, pp. 1389-1405, 2008.

2. A. N. Vgontzas, A. Kales, "Sleep and its disorders", Annu Rev Med, vol. 50, pp. 387-400, 1999.

3. T. Rantonen, J. Jalonen, J. Grönlund, K. Antila, D. Southall, I. Välimä$\mathrm{ki}$, "Increased amplitude modulation of continuous respiration precedes sudden infant death syndrome -Detection by spectral estimation of respirogram", Early Human Development, vol. 53, no. 1, pp. 53-63, 1998.

4. F. Q. AL-Khalidi, R. Saatchi, D. Burke, H. Elphick, S. Tan, "Respiration rate monitoring methods: A review", Pediatr Pulmonol, vol. 46, no. 6. pp. 523-529, 2011.

5. W. Karlen, C. J. Brouse, E. Cooke, J. M. Ansermino, G. A. Dumont, "Respiratory rate estimation using respiratory sinus arrhythmia from photoplethysmography", Annual International Conference of the IEEE Engineering in Medicine and Biology Society, 2011.

6. A. Gaucher, D. Frasca, O. Mimoz, B. Debaene, "Accuracy of respiratory rate monitoring by capnometry using the Capnomask ${ }^{\circledR}$ in extubated patients receiving supplemental oxygen after surgery", Br J Anaesth, vol. 108, no. 2, pp. 316-320, 2012.

7. Y. Nam, B. A. Reyes, K. H. Chon, "Estimation of Respiratory Rates Using the Built-in Microphone of a Smartphone or Headset", IEEE J Biomed Health Inform, vol. 20, no. 6. pp. 1493-1501, 2016.

8. P. Corbishley, E. Rodríguez-Villegas, "Breathing detection: Towards a miniaturized, wearable, battery-operated monitoring system", IEEE Trans Biomed Eng, vol. 55, no. 1, pp. 196-204, 2008.

9. J. Hernandez, D. McDuff, R. Picard, "BioWatch: Estimation of Heart and Breathing Rates from Wrist Motions", EAl Endorsed Transactions on Pervasive Health and Technology, vol. 15, no. 3, 2015.

10. M. Bartula, T. Tigges, J. Muehlsteff, "Camera-based system for contactless monitoring of respiration", $35^{\text {th }}$ Annual International Conference of the IEEE Engineering in Medicine and Biology Society (EMBC), 2013, pp. 2672-2675.

11. K. Nakajim, Y. Matsumoto, T. Tamura, "Development of real-time image sequence analysis for evaluating posture change and respiratory rate of a subject in bed", Physiol Meas, vol. 22, no. 3, pp. 21-28, 2001.

12. K. S. Tan, R. Saatchi, H. Elphick, D. Burke, "Real-time vision based respiration monitoring system", 7th International Symposium on Communication Systems, Networks \& Digital Signal Processing (CSNDSP 2010), pp. 770-774, 2010.

13. A. Lazaro, D. Girbau, R. Villarino, "Analysis of Vital Signs Monitoring Using an IR-UWB Radar", Prog Electromagn Res, vol. 100, pp. 265284, 2010.

14. A. D. Droitcour, O. Boric-Lubecke, G. T. A. Kovacs, "Signal-to-Noise Ratio in Doppler Radar System for Heart and Respiratory Rate Measurements", IEEE Transactions on Microwave Theory and Techniques, vol. 57, no. 10. pp. 2498-2507, 2009.

15. C. Li, J. Ling, J. Li, J. Lin, "Accurate doppler radar noncontact vital sign detection using the RELAX algorithm", IEEE Transactions on Instrumentation and Measurement, vol. 59, no. 3, pp. 687-695, 2010.

16. M. Ascione, A. Buonanno, M. D'Urso, L. Angrisani, R. S. Lo MorielIo, "A new measurement method based on music algorithm for through-the-wall detection of life signs", IEEE Transactions on Instrumentation and Measurement, vol. 62, no. 1. pp. 13-26, 2013.

17. C. Li, J. Lin, "Recent advances in Doppler radar sensors for pervasive healthcare monitoring", Asia-Pacific Microwave Conference (APMC), 2010, pp. 283-290.

18. R. Schmidt, "Multiple emitter location and signal parameter estimation", IEEE Trans. Antennas Propag, vol. 34, no. 3, pp. 276-280, Mar. 1986.

19. N. Patwari, J. Wilson, S. Ananthanarayanan, S. K. Kasera, D. R. Westenskow, "Monitoring breathing via signal strength in wireless networks", IEEE Transactions on Mobile Computing, vol. 13, no. 8, pp. 1774-1786, 2014.

20. X. Liu, J. Cao, S. Tang, J. Wen, P. Guo, "Contactless Respiration Monitoring Via Off-the-Shelf WiFi Devices", IEEE Transactions on Mobile Computing, vol. 15, no. 10. pp. 2466-2479, 2016. 
21. O. Kaltiokallio, H. Yiğitler, R. Jäntti, and N. Patwari, "Non-invasive respiration rate monitoring using a single COTS TX-RX pair," IPSN 2014 - Proceedings of the $13^{\text {th }}$ International Symposium on Information Processing in Sensor Networks, 2014, pp. 59-69,

22. F. Adib, H. Mao, Z. Kabelac, D. Katabi, R. C. Miller, "Smart Homes that Monitor Breathing and Heart Rate", Proceedings of the $33^{\text {rd }} \mathrm{An}$ nual ACM Conference on Human Factors in Computing Systems, pp. 837-846, 2015.

23. J. Liu, Y. Wang, Y. Chen, J. Yang, X. Chen, J. Cheng, "Tracking Vital Signs During Sleep Leveraging Off-the-shelf WiFi," Proceedings of the $16^{\text {th }}$ ACM International Symposium on Mobile Ad Hoc Networking and Computing, pp. 267-276, 2015.

24. H. Abdelnasser, K. A. Harras, M. Youssef, "UbiBreathe: A Ubiquitous non-invasive WiFi-based Breathing Estimator", Proceedings of the $16^{\text {th }}$ ACM International Symposium on Mobile Ad Hoc Networking and Computing, pp. 277-286, 2015

25. R. Ravichandran, E. Saba, K. Y. Chen, M. Goel, S. Gupta, S. N. Patel, "WiBreathe: Estimating respiration rate using wireless signals in natural settings in the home", IEEE International Conference on Pervasive Computing and Communications (PerCom), 2015, pp. 131-139, 2015.

26. C. Wu, Z. Yang, Z. Zhou, X. Liu, Y. Liu, J. Cao, “Non-invasive detection of moving and stationary human with WiFi," IEEE Journal on
Selected Areas in Communications, vol. 33, no. 11, pp. 2329-2342, 2015.

27. H. Wang, D. Zhang, J. Ma, Yasha Wang, Yuxiang Wang, D. Wu, T. Gu, B. Xie, "Human respiration detection with commodity wifi devices: Do User Location and Body Orientation Matter?", Proceedings of the 2016 ACM International Joint Conference on Pervasive and Ubiquitous Computing, 2016, pp. 25-36.

28. C. Uysal and T. Filik, "Contactless respiration rate estimation using MUSIC algorithm," in Electrical and Electronics Engineering (ELECO), 2017 10th International Conference on, 2017, pp. 606-610.

29. Z. Yang, Z. Zhou, Y. Liu, "From RSSI to CSI", ACM Computing Surveys (CSUR), vol. 46, no. 2, pp. 1-32, 2013.

30. S. Venkatesh, C. R. Anderson, N. V. Rivera, R. M. Buehrer, "Implementation and analysis of respiration-rate estimation using impulse-based UWB", IEEE Military Communications Conference, 2005.

31. C. Lowanichkiattikul, M. Dhanachai, C. Sitathanee, S. Khachonkham, and P. Khaothong, "Impact of chest wall motion caused by respiration in adjuvant radiotherapy for postoperative breast cancer patients," Springerplus, vol. 5, no. 1, pp. 1-8, 2016.

32. L. Davies and U. Gather, "The identification of multiple outliers", J Am Stat Assoc, vol. 88, no. 423, pp. 782-792, 1993.

Can Uysal received his B.S. degree in Electronics and Communications Engineering from Kocaeli University in 2009 and his M.S. degree from the Electrical and Electronics Engineering from Anadolu University in 2015. He is currently a Ph.D. candidate at Anadolu University in department of Electrical and Electronics Engineering. His research interests include sensor array processing, RF-sensing, biomedical applications of signal processing.

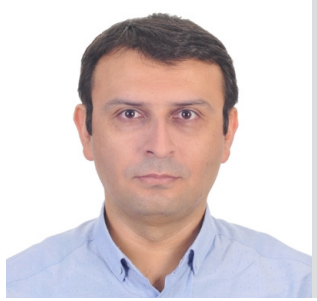

Tansu Filik received the B.S. degree from the Anadolu University in 2002, the integrated M.S. and Ph.D. degree from the Middle East Technical University (METU) in 2010, all in electrical engineering. From 2002 to 2010, he was withSensor Array and Multichannel Signal Processing (SAM) Group of METU. From 2010 to 2012, he was with Aselsan Inc., at Radar, Electronic Warfare and Intelligence Systems Division. Currently, he is with Anadolu University at Department of Electrical Engineering. His research interest includes statistical signal processing, array signal processing, signal processing for communications and renewable energy. He is the founder of the Sensor Array Signal Processing Laboratory in the Department of Electrical and Electronics Engineering. 